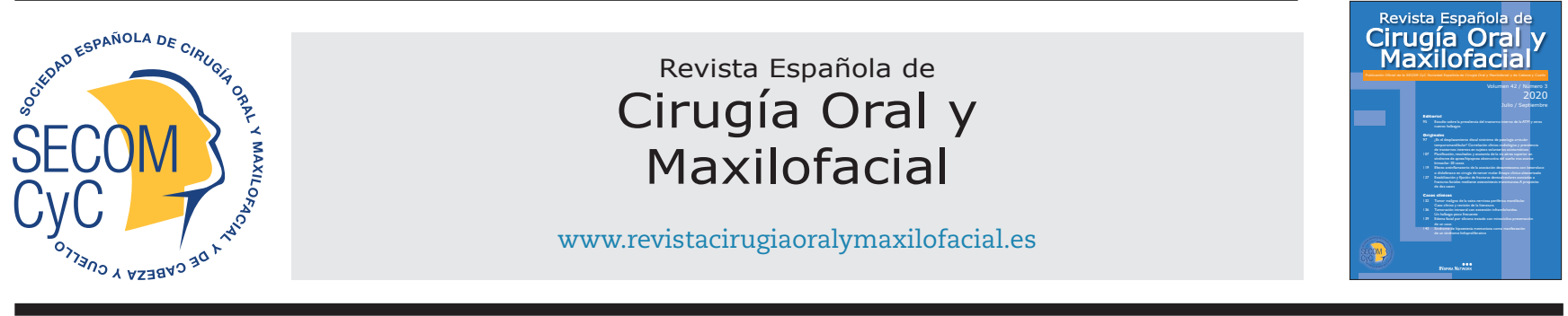

\title{
Original
}

\section{Estabilización y fijación de fracturas dentoalveolares asociadas a fracturas faciales mediante osteosíntesis transmucosa. A propósito de dos casos}

\author{
Ilich Vargas*, Felipe Gutiérrez, Eduardo Navia, Luis Edgardo González, \\ Christian Pedemonte y Patricio Celedón Olivera
}

Unidad de Cirugía Maxilofacial, Hospital Clínico Mutual de Seguridad, Santiago de Chile, Chile

\section{INFORMACIÓN DEL ARTÍCULO}

\section{Historia del artículo:}

Recibido: 19 de septiembre de 2017

Aceptado: 31 de enero de 2019

Palabras clave:

Placas transmucosas, osteosintesis transmucosa, fracturas conminutas, fracturas dentoalveolar, trauma maxilofacial.

\section{R E S U M E N}

Objetivos: Evaluar la estabilidad de osteosíntesis transmucosas, en casos de fracturas conminutas dentoalveolares, evitando la desperiostización de los fragmentos, para así mantener la vascularización de estos, favoreciendo la regeneración y pronóstico de las piezas dentarias y los segmentos óseos.

Materiales y métodos: Se realizó osteosíntesis transmucosa con placas de perfil delgado $(0,5$ y 0,7 mm dePuy Synthes - Matrix Midface Kit) para la reducción de fracturas de procesos alveolares en 3 localizaciones de 2 pacientes que en asociación presentaban fracturas faciales complejas tratadas con reducción abierta y fijación interna convencional. Además se instalaron férulas con alambres de acero de 0,5 $\mathrm{mm}$ en los dientes involucrados.

Resultados: Se logró una correcta reducción, corrección y fijación de los fragmentos y la conservación de dientes involucrados en las 3 localizaciones, observable tanto clínicamente como en las tomografías computarizadas a los 3 y 7 meses. Además, se retiraron las placas de osteosíntesis de forma ambulatoria y mediante anestesia local.

Conclusiones: La estabilización y fijación con placas transmucosas favorece la consolidación de los segmentos afectados sin afectar el aporte vascular. Sin embargo, este método puede considerarse una medida de asistencia a la reducción abierta con fijación interna, que sigue siendo la medida de primera elección.

\footnotetext{
*Autor para correspondencia:

Correo electrónico: ilich.vargas@gmail.com (Ilich Vargas).
}

DOI: $10.20986 /$ recom.2020.1045/2019

1130-0558/@ 2020 SECOM. Publicado por Inspira Network. Este es un artículo Open Access bajo la licencia CC BY-NC-ND (http:// creativecommons.org/licenses/by-nc-nd/4.0/). 


\section{Stabilization and fixation of dentoalveolar fractures asociated with facial fractures across transmucosal osteosynthesis. A propos of two cases}

\section{A B S T R A C T}

\section{Keywords:}

Transmucosal plates, transmucosal osteosynthesis, comminuted fractures, dentoalveolar fractures, maxillofacial trauma.
Objectives: To assess the stability of transmucosal osteosynthesis in cases of comminuted dentoalveolar fractures, avoiding the deperiostization of fragments to maintain their vascularization, favoring the regeneration and prognosis of dental pieces and bone segments. Materials and methods: Transmucosal osteosynthesis with low-profile plates ( 0.5 and $0.7 \mathrm{~mm}$ of Puy Synthes - Matrix Midface Kit) was performed to reduce fractures of alveolar processes in 3 locations of 2 patients, who in association had complex facial fractures treated with open reduction and conventional internal fixation. Furthermore, splints with $0.5 \mathrm{~mm}$ steel wires were installed on the teeth involved.

Results: A correct reduction, correction, and fixation of the fragments and the preservation of teeth involved in the 3 locations, observable both clinically and in CT scans at 3 and 7 months was achieved. Besides, the osteosynthesis plates were removed on an outpatient basis and by local anesthesia.

Conclusions: Stabilization and fixation with transmucosal plates favors the consolidation of the affected segments without affecting the vascular supply. However, this method can be considered an open reduction assistance measure with internal fixation, which remains the first-choice measure.

\section{INTRODUCCIÓN}

El trauma facial es en la actualidad una entidad patológica de alta prevalencia a nivel mundial, constituyéndose como un problema de salud pública de elevada importancia ${ }^{1,2}$. Por otra parte, el tratamiento del trauma facial incluye el manejo de tejidos blandos (piel y mucosas) y tejidos duros (tejido óseo y estructuras dentoalveolares), según sea la naturaleza de las lesiones. Hoy en día, el tratamiento temprano mediante reducción abierta y fijación interna se considera la conducta terapéutica de predilección en la mayoría de los casos de fracturas del macizo facial.

La reducción abierta y fijación interna tradicionalmente requieren de la exposición de los focos fracturarios a través de abordajes o accesos quirúrgicos y su reducción anatómica directa para posteriormente instalar material de osteosíntesis de acuerdo con cada situación y diagnóstico. Sin embargo, en fracturas conminutas con fragmentos pequeños, la diéresis del paquete mucoperióstico podría producir un perjuicio para mantener fragmentos óseos, interrumpiendo el aporte vascular, la llegada de nutrientes y la capacidad inmunológica, aumentando la tendencia a la necrosis $\mathrm{s}^{3-5}$ y generando defectos óseos que posteriormente requerirán cirugía complementaria para corregirlos. Es por este motivo que planteamos que el manejo conservador y la posibilidad de evitar la desperiostización en algunos casos podrían considerarse como una medida de tratamiento que permita favorecer el pronóstico de la preservación dentoalveolar para el paciente. El presente trabajo busca plantear una alternativa de tratamiento para fracturas dentoalveolares asociadas a fracturas faciales complejas, describiendo el manejo y resultados de dos casos, los cuales fueron tratados mediante la instalación de placas de osteosíntesis transmucosa de perfil delgado y tornillos monocorticales, además de la instalación de férulas semirrígidas.

\section{MATERIAL Y MÉTODOS}

Se realizó la técnica de instalación de osteosíntesis transmucosa con placas de perfil delgado $(0,5$ y 0,7 $\mathrm{mm}$ dePuy Synthes - Matrix Midface Kit) en dos pacientes que presentaban múltiples fracturas faciales, las cuales fueron tratadas mediante reducción abierta y fijación interna tradicional. Ambos pacientes presentaron compromiso dentoalveolar en tres segmentos diferentes en total. En el paciente $n .^{\circ} 1$-con diagnósticos de fractura parasinfisiaria, condilar y fractura del proceso alveolar a nivel maxilar y mandibular (Figura 1)-, además de la reducción abierta y la fijación interna rígida de las fracturas mandibulares, se colocaron dos placas de osteosíntesis transmucosa, una a nivel maxilar aislada y otra a nivel mandibular, ambas con tornillos monocorticales de $6 \mathrm{~mm}$, procurando fijar el segmento de tejido óseo alveolar sin dañar las raíces de los dientes involucrados. Al finalizar el cierre de las heridas quirúrgicas se colocó una férula alámbrica de acero redondo de 0,5 $\mathrm{mm}$ en los dientes involucrados. En el paciente $\mathrm{n} .^{\circ} 2$-con diagnóstico de fractura LeFort II asociada a fractura media palatina y fractura alveolar de segmento anterior maxilar- se colocó una placa de perfil delgado con tornillos monocorticales a nivel de la fractura dentoalveolar asociada a la fractura media palatina (Figura 2), y posteriormente al cierre de las heridas se realizó la ferulización de los dientes involucrados. En ambos casos se colocaron estas placas con el objetivo de complementar la reducción y osteosíntesis tradicional de las fracturas faciales asociadas. 


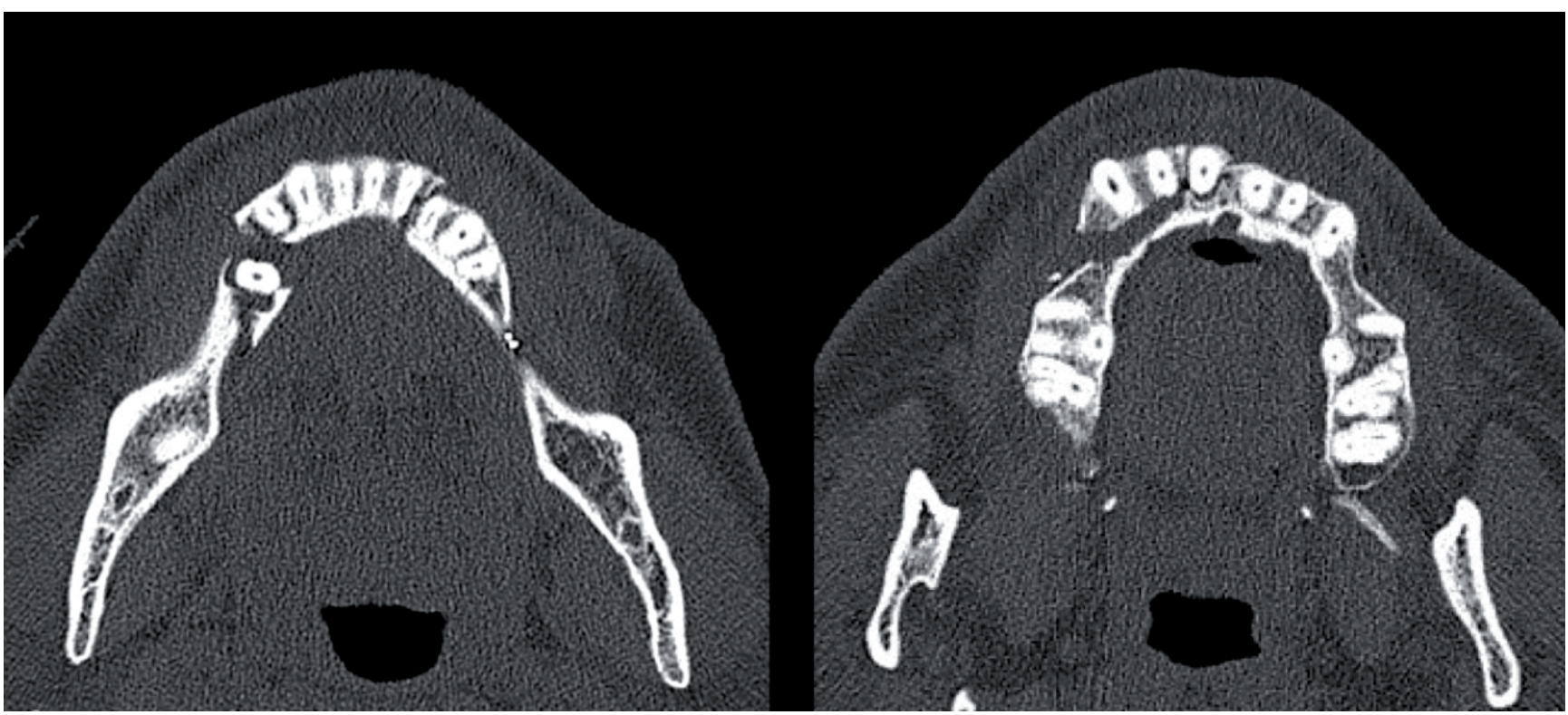

Figura 1. Paciente n. ${ }^{\circ}$ : diagnósticos de fractura parasinfisiaria, condilar y fractura del proceso alveolar a nivel maxilar y mandibular.

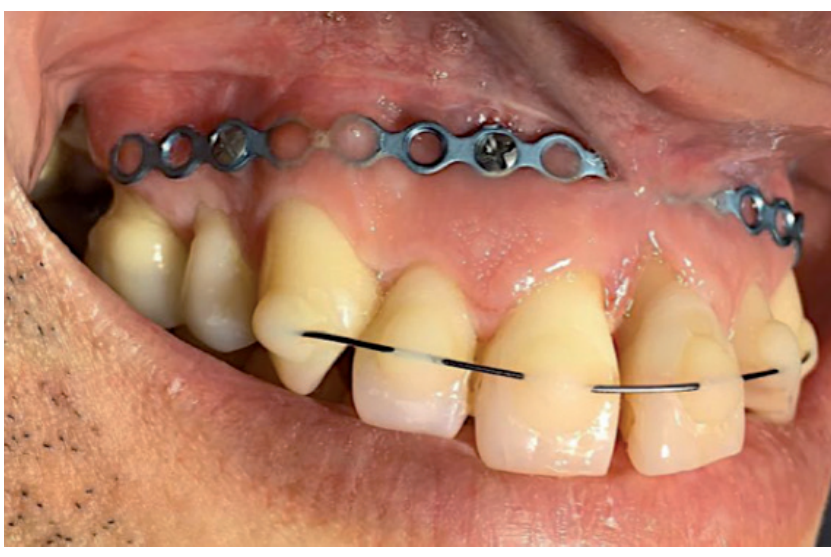

Figura 2. Disposición de los tornillos.

La técnica se llevó a cabo con la colocación de placas a nivel del límite mucogingival de la zona afectada procurando que su extensión se prolongara hasta el tejido estable con al menos tres a cuatro orificios. Los tornillos fueron dispuestos en aquellos segmentos que presentaban movilidad e inestabilidad, y en los extremos estables se instalaron al menos dos tornillos, siempre evitando dañar los dientes involucrados mediante la visualización y palpación de su proyección (Figura 2).

\section{RESULTADOS}

En uno de los tres sitios en que se colocaron placas transmucosas se produjo un proceso necrótico de la mucosa circundante a la placa de osteosíntesis; sin embargo, se logró una correcta recuperación de los tejidos bajo la indicación de aseos locales con colutorios de digluconato de clorhexidina al 0,12 \% de concentración. En los otros dos sitios las condiciones de la mucosa fueron concordantes con una adecuada salud de encía y mucosa.

En las tres localizaciones las fracturas presentaron una adecuada reducción, estabilización, fijación y consolidación, siendo posible la conservación de las piezas dentarias involucradas. En las tomografías computarizadas de control a medio y largo plazo (7 y 3 meses, respectivamente) es posible observar un posicionamiento adecuado de los segmentos alveolares comprometidos y una consolidación ósea óptima (Figura 3). Por otra parte, en ambos pacientes se retiraron los elementos de osteosíntesis de forma ambulatoria y con administración de anestésicos locales sin presentar complicaciones posteriores. Además, aparentemente, en el paciente n. ${ }^{\circ} 2$ la placa de osteosíntesis transmucosa actuó como un estabilizador en sentido sagital (que no garantiza la reducción anatómica pero sí aporta beneficio al tratamiento).

\section{DISCUSIÓN}

Si bien la reducción abierta y fijación interna es considerada actualmente como la vía de manejo más adecuada para la mayoría de fracturas del macizo facial, existen situaciones en que la desperiostización de los fragmentos podría inducir su necrosis o la pérdida por interrupción de la vascularización periostal ${ }^{2,4,5}$, y es por esto que, bajo nuestra perspectiva, sería ideal apuntar a buscar metodologías de mínima invasión para el manejo de algunos casos de compromiso alveolar.

Múltiples estudios describen y reconocen la importancia de la irrigación y de las células osteoblásticas provenientes del periostio y la cubierta mucosa para la adecuada migra- 


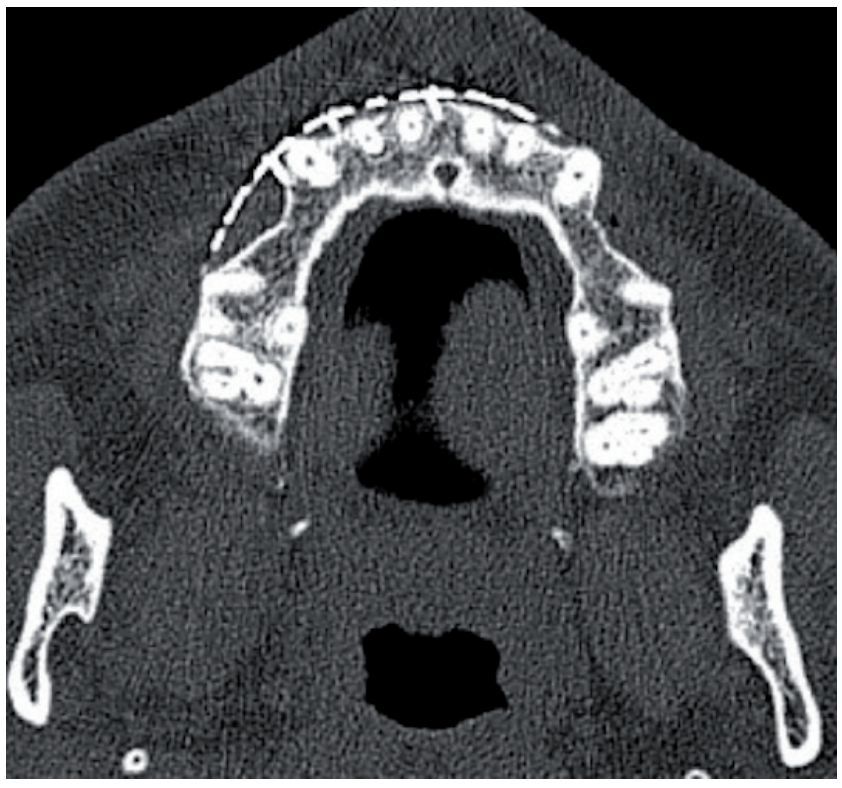

Figura 3. Tomografías computarizadas de control a medio y largo plazo (7 y 3 meses, respectivamente). Se observa un posicionamiento adecuado de los segmentos alveolares comprometidos y una consolidación ósea óptima.

ción celular y la subsecuente consolidación de la fractura ${ }^{6-8}$; sin embargo, la bibliografía respecto a este tipo de técnicas en fracturas alveolares es escasa. Por otra parte, en torno a temas de controversia en cirugía maxilofacial, algunos autores plantean la reducción cerrada como método de elección en cierto tipo de fracturas mandibulares conminutas y con compromiso de tejidos blandos, justamente con la finalidad de preservar la bolsa perióstica, donde los mismos fragmentos óseos actúan como injertos, que posteriormente osifican, manteniendo la continuidad y función del segmento fracturado ${ }^{9-11}$.

En 2005, Kanagaraja y cols. y en 2011 Wood y cols. publicaron casos y notas técnicas relativos a la utilización de placas de osteosíntesis transmucosa para lograr la fijación y estabilización en casos de fracturas conminutas y en mandíbulas edéntulas. Reportaron principalmente un buen rendimiento de la técnica, además de la necrosis del tejido circundante como una de las complicaciones transitorias frecuentes en este tipo de tratamiento, consecuencia de la compresión de los sistemas de osteosíntesis, lo que generaba isquemia y necrosis de la mucosa circundante ${ }^{12,13}$. Respecto a esto, si bien nuestros pacientes no presentaban edentulismo total ni conminución local, la técnica fue aplicable y exitosa para el tratamiento de las fracturas dentoalveolares asociadas a otras fracturas faciales. En relación con la necrosis del tejido blando circundante, esta se presentó en el segundo caso (fractura alveolar asociada a fractura media palatina) y fue tratada exitosamente con aseos de clorhexidina al 0,12\%. Tras la retirada de la placa, curó de forma normal, sin complicaciones ni secuelas posteriores. Debido a esta complicación nace el planteamiento de puentear las zonas de compresión de las placas sobre la mucosa, utilizando placas bloqueadas, cuyo contacto con la mucosa sería menor, lo cual podría evitar la mencionada complicación.

Por su parte, el manejo tradicional de las fracturas dentoalveolares propone un tratamiento cerrado a fin de no interrumpir el aporte vascular local y la colocación de férulas semirrígidas o rígidas tales como arcos metálicos, alambres de acero y splints acrílicos durante 4 semanas o más, cuya objetivo es fijar los dientes del segmento afectado a las piezas vecinas; además de controlar el trauma oclusal mediante modificaciones de la dieta (régimen blando) o tratando mecánicamente el contacto dentario ${ }^{14-16}$. Sin embargo, bajo nuestra apreciación, esta medida no logra una estabilidad tridimensional total y una fijación óptima del proceso alveolar afectado, sobre todo en aquellos casos donde coexisten fracturas dentoalveolares y fracturas faciales. Es por estas razones que la instalación de placas y tornillos por vía transmucosa, en conjunto con la ferulización dental semirrígida, podría estabilizar los segmentos alveolares de forma más adecuada sin interrumpir la irrigación de los tejidos afectados, lo que favorecería su consolidación.

Podemos concluir que la estabilización y fijación mediante la colocación de placas de osteosíntesis transmucosa en el caso de fracturas dentoalveolares favorece la adecuada consolidación de los segmentos afectados sin afectar el aporte vascular. Sin embargo, bajo nuestra perspectiva, en el trauma facial, la reducción abierta con fijación interna sigue siendo la medida de primera elección, y la colocación de placas transmucosas podría considerarse solo una medida de asistencia al método tradicional.

\section{B I B L I O G R A F Í A}

1. Boffano P, Roccia F, Zavattero E, Dediol E, Ugleši冈V, Kova囚ið Ž, et al. European Maxillofacial Trauma (EURMAT) project: A multicentre and prospective study J Craniomaxillofac Surg. 2015;43(1):62-70. DOI: 10.1016/j.jcms.2014.10.011.

2. Gonzalez E, Pedemonte C, Vargas I, Lazo D, Pérez H, Canales M, et al. Facial fractures in a reference center for Level I Traumas. Descriptive study. Rev Esp Cir Oral y Maxilofac (English Edition). 2015;37(2):65-70. DOI: 10.1016/j.maxile.2013.09.001.

3. Smith B, Teenier T. Treatment of conminuted mandibular fractures by open reduction and rigid fixation. J Oral Maxillofac Surg. 1996;54(3):328-31. DOI: 10.1016/s0278-2391(96)90754-5.

4. Abreu MER, Viegas VN, Ibrahim D, Valiati R, Heitz C, Pagnoncelli RM, et al. Treatment of comminuted mandibular fractures: A critical review. Med Oral Patol Oral Cir Bucal. 2009;14(5):E24751.

5. Madsen MJ, Haug RH, Christensen BS, Aldridge E. Management of atrophic mandible fractures. Oral Maxillofacial Surg Clin N Am. 2009;21(2):175-83. DOI: 10.1016/j.coms.2008.12.006.

6. Colnot C. Skeletal cell fate decisions within periosteum and bone marrow during bone regeneration. J Bone Miner Res. 2009;24(2):274-82. DOI: 10.1359/jbmr.081003.

7. Diaz-Flores L, Gutierrez R, Lopez-Alonso A, Gonzalez R, Varela H. Pericytes as a supplementary source of osteoblasts in periosteal osteogenesis. Clin Orthop Relat Res. 1992;275:280-6.

8. Bruce RA, Ellis E. The Second Chalmers J. Lyons Academy Study of Fractures of the Edentulous Mandible. J Oral Maxillofac Surg. 1993;51(8):904-11. DOI: 10.1016/s0278-2391(10)80113-2.

9. Bradley JC. Age changes in the vascular supply of the mandible. Br Dent J. 1972;132(4):142-4. DOI: 10.1038/sj.bdj.4802812. 
10. Lin Z, Fateh A, Salem DM, Intini G. Periosteum: Biology and applications in craniofacial bone regeneration. J Dent Res. 2014;93(2):109-16. DOI: 10.1177/0022034513506445.

11. Chrcanovic BR. Open versus closed reduction: comminuted mandibular fractures. Oral Maxillofac Surg. 2013;17(2):95-104 DOI: 10.1007/s10006-012-0349-2.

12. Wood GA, Campbell DF, Greene LE. Transmucosal fixation of the fractured edentulous mandible. Int J Oral Maxillofac Surg. 2011;40(5):549-52. DOI: 10.1016/j.ijom.2010.10.027.

13. Kanagaraja S, Kashani H, Zellin G. Fixation of Comminuted alveolar ridge fracture after severe trauma by application of ti-osteosynthesis plates on soft tissue: a technical note. J Oral
Maxillofac Surg. 2005;63(5):720-2. DOI: 10.1016/j. joms.2004.06.063.

14. Olynik CR, Gray A, Sinada GG. Dentoalveolar trauma. Otolaryngol Clin N Am. 2013;46(5):807-23. DOI: 10.1016/j. otc.2013.06.009.

15. Lim L, Sirichai P. Bone fractures: assessment and management. Aust Dent J. 2016;61 Suppl 1:74-81. DOI: 10.1111/adj.12399.

16. Flores MT, Andersson L, Andreasen J, Bakland LK, Malmgren B, Barnett F, et al. Guidelines for the management of traumatic dental injuries. I. Fractures and luxations of permanent teeth. Dent Traumatol. 2007;23(2):66-71. DOI: 10.1111/j.1600-9657.2007.00592.x. 